\title{
Room Temperature CO Dissociation on Selective Edges of Gold Nanoparticles
}

\author{
Wei-Chang D. Yang ${ }^{1,2}$, Canhui Wang ${ }^{1,2}$ and Renu Sharma ${ }^{1}$ \\ 1. Center for Nanoscale Science and Technology, National Institute of Standards and Technology, \\ Gaithersburg, Maryland 20899 (USA) \\ 2. Maryland NanoCenter, University of Maryland, College Park, MD 20742 (USA)
}

Au nanoparticles on oxide supports have drawn great interest as catalysts because of their potential for reactions such as low temperature $\mathrm{CO}$ oxidation[1]. Both experimental and theoretical results have suggested that adsorption of $\mathrm{CO}$ molecules on $\mathrm{Au}$ surfaces and the resulting catalytic activity are affected by the coordination number of the surface $\mathrm{Au}$ atoms[1]. However, direct evidence of the locations of $\mathrm{CO}$-adsorbing sites on the nanoparticle surface, which may be catalytically active sites, is still missing.

In the present work, we take advantage of the plasmonic behavior of Au nanoparticles to measure the energy shifts in localized surface plasmon resonance (LSPR) energy as a function of gas environment using an environmental scanning transmission electron microscope equipped with a monochromated field-emission gun. Optical methods that are used so far to measure LSPR energies have spatial resolution limited to $\approx 100 \mathrm{~nm}$. We use electron energy loss spectrum (EELS) imaging, at high energy and spatial resolution, to map the LSPR intensities of an Au nanoparticle with a triangular bi-prism shape (Fig. 1a). We identify the location of CO adsorption on the Au nanoparticle from the spectral shift of the LSPR energy[2]. The LSPR peak (Fig. 1b) blue-shifts $(\approx 0.05 \mathrm{eV})$ in $\mathrm{CO}$ environment $\left(\mathrm{P}_{\mathrm{CO}}=110\right.$ $\mathrm{Pa}$ ) with respect to vacuum at the sides of $\mathrm{Au}$ triangular nanoparticles. The sides of triangular nanoparticles consist of ledges that are terminated by the $\{311\}$ planes of $\mathrm{Au}$, as identified from the diffraction data collected by tilting the particle. The subsequent core-loss EELS revealed the existence of $\mathrm{C} \mathrm{K}$-edge corresponding to the amorphous carbon that deposited at the same location (Fig. 1c). The green region in Fig. 1d, which is associated with the measured $\mathrm{C}$ K-edge intensities, indicates the location of amorphous carbon. While the LSPR peak shifted back to its original value after CO was evacuated, the amorphous carbon remained on the ledges, indicating that the carbon signal is not from the CO and the LSPR energy shift is not due to carbon deposition. It is interesting to note that the clean surface of $\mathrm{TiO}_{2}$ support, which does not display any LSPR signal (Fig. 1a), shows no sign of amorphous carbon deposits (Fig. 1d), suggesting that i) the carbon deposition is not caused by carbonaceous contamination, ii) the LSPR of $\mathrm{Au}$ is essential for carbon deposition, and iii) therefore, carbon is deposited by the room temperature dissociation of $\mathrm{CO}$. We believe that our results can be compared with the recently reported dissociation of $\mathrm{H}_{2}$, facilitated by the hot electrons generated from the non-radiative decay of LSPR[3]. Dissociation energies of CO molecules may be lowered as a result of CO adsorption on selective Au surfaces/ledges as reported for Fe $\{100\}$ surfaces[4]. The locations that are selective for amorphous carbon deposition may correspond to the active sites that display decreased dissociation barriers and enable the hot electron transfer.

Our result suggests that $\mathrm{CO}$ is adsorbed preferentially on the ledges of the Au nanoparticles. More interestingly, we discover that LSPR can dissociate CO molecules at room temperature as evidenced by the deposition of amorphous carbon on the same ledges. 


\section{References:}

[1] N Lopez et al., J. Catal. 223 (2004) p. 232.

[2] GM Lari et al., Phys. Chem. Chem. Phys. 17 (2015) p. 23236.

[3] S Mukherjee et al., Nano Lett. 13 (2012) p. 240.

[4] DE Jiang and EA Carter, Surf. Sci. 570 (2004) p. 167.

[5] WD Yang and C Wang acknowledge support under the Cooperative Research Agreement between the University of Maryland and the National Institute of Standards and Technology Center for Nanoscale Science and Technology, Award 70NANB14H209, through the University of Maryland.
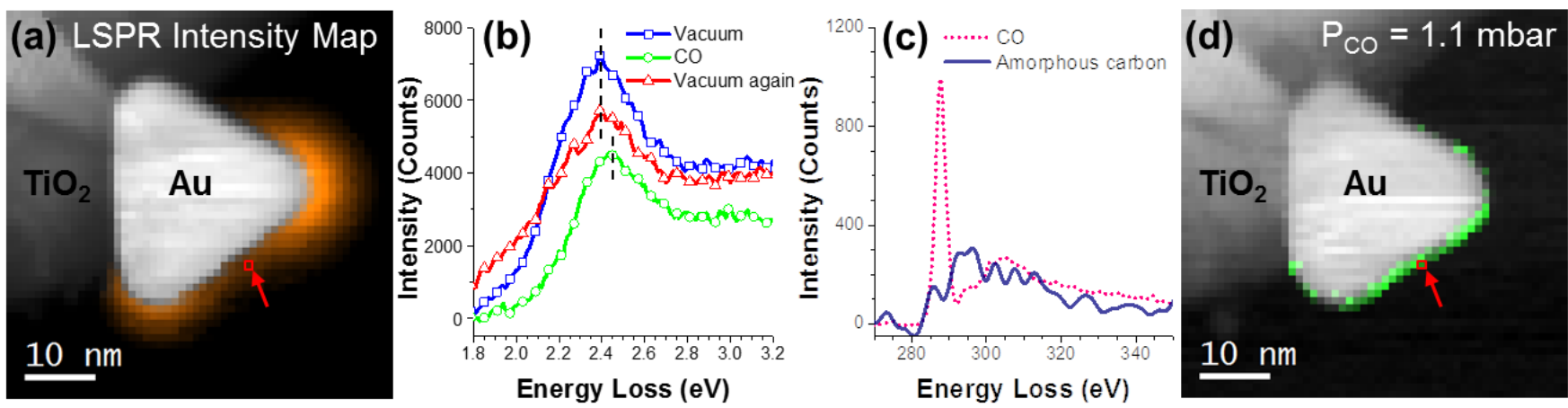

Figure 1. a) LSPR intensity map (orange) overlaid on the annular dark field (ADF) image of $\mathrm{Au} / \mathrm{TiO}_{2}$ catalyst in vacuum, also noted that the LSPR intensity at the top corner of the Au nanoparticle is attenuated by plural scattering due to the increased sample thickness as Au nanoparticle overlaps with $\mathrm{TiO}_{2}$ support, b) low-loss spectra of LSPR in vacuum, $\mathrm{CO}$ environment and vacuum again, respectively, from the denoted pixel in a), c) core-loss spectra of $\mathrm{CO}$ background and deposited amorphous carbon, d) amorphous carbon map (green) overlaid with the $\mathrm{ADF}$ image of $\mathrm{Au} / \mathrm{TiO}_{2}$ catalyst in $\mathrm{CO}$ environment. 